\title{
Scattering in the vicinity of relativistic jets: A method for constraining jet parameters
}

\author{
C. K. Cramphorn ${ }^{1,3}$, S. Yu. Sazonov ${ }^{1,2}$, and R. A. Sunyaev ${ }^{1,2}$ \\ ${ }^{1}$ Max-Planck-Institut für Astrophysik, Karl-Schwarzschild-Str. 1, 85740 Garching bei München, Germany \\ 2 Space Research Institute, Russian Academy of Sciences, Profsoyuznaya 84/32, 117997 Moscow, Russia \\ 3 Kanzlei Dr. Volker Vossius, Geibelstr. 6, 81679 München, Germany
}

Received 1 February 2004 / Accepted 11 March 2004

\begin{abstract}
Relativistic jets of radio loud active galactic nuclei (AGN) produce highly directed, intense beams of radiation. A fraction of this beamed radiation scatters on the thermal plasma generally surrounding an AGN. The morphology of the scattered emission can thus provide constraints on the physical properties of the jet. We present a model to study the feasibility of constraining the parameters of a jet, especially its inclination angle and bulk Lorentz factor in this way. We apply our model to the well studied jet of M 87 and the surrounding diffuse gas and find that the observational limits of the surface brightness measured in the region of the putative counterjet provide the tightest constraints on the jet parameters consistent with constraints derived by other methods. We briefly discuss the applicability of our model to other sources exhibiting relativistic motions.
\end{abstract}

Key words. scattering - galaxies: jets - galaxies: individual: M 87 - galaxies: clusters: general - radio continuum: general

\section{Introduction}

Giant ellipticals in the central parts of clusters of galaxies often harbour an active galactic nucleus (AGN) which is believed to be powered by a supermassive black hole. X-ray observations have revealed that these AGN are surrounded by an atmosphere consisting of a hot and tenuous thermal plasma that fills the intracluster space. The optical depth for Thomson scattering due to the free electrons present in this plasma reaches values of the order of $10^{-3}-10^{-2}$. Therefore, up to one per cent of the luminosity of an AGN radiating in such an environment will be scattered on the free thermal electrons and this scattered radiation will be highly polarised (Sunyaev 1982).

A rather interesting situation arises in the case of an AGN powering a radio galaxy through a pair of relativistic jets which emit synchrotron radiation. The radiation produced by the relativistic particles gyrating along the magnetic fields of the jet is strongly beamed, i.e. the radiation field is highly anisotropic in the vicinity of the jets, due to well known relativistic effects.

The free electrons present in the hot thermal plasma, on the other hand, are distributed in a more or less spherically symmetrical way around the AGN. The electrons located close to the jet are exposed to the largest radiative flux and, therefore, will be the strongest sources of scattered radiation. Thus, the surface brightness profile of the electron scattered component should reflect the anisotropy of the jet emission and could provide information about the intrinsic jet properties.

Send offprint requests to: C. K. Cramphorn, e-mail: conrad.cramphorn@vovo.de
Combining this information with knowledge about the density distribution of the scattering gas, which for instance is obtainable through X-ray observations, might enable one to gain information about such parameters as the inclination angle of the jet axis towards the line of sight and the bulk Lorentz factor of the jet flow. The feasibility of this method will be studied in this paper.

The "standard model" of extragalactic jets (Blandford \& Rees 1974; Scheuer 1974) describes such objects as intrinsically symmetric structures. The different appearances of the jet pointing towards the observer in comparison with the one pointing away from the observer, the so-called counterjet, stems primarily from the orientation of the oppositely directed axes of these jets with respect to the observer. In the case of a small inclination angle and a large Lorentz factor the counterjet might be rendered practically invisible, as is presumably the case in e.g. M 87, offering only indirect evidence for its existence in the form of e.g. corresponding hotspots and radio lobes.

With respect to the question addressed in this paper, however, it is important to note that the electrons being illuminated by the counterjet are exposed to and scatter as much radiation as the electrons located on the side of the jet. The only difference obviously being that the photons scattered by electrons located on the side of the counterjet have to travel larger distances in order to reach the distant observer than photons scattered by electrons located on the side of the jet.

The observational problem related to the detection of the discussed effect against the "background" produced by other sources of radio emission, e.g. the jets themselves, radio lobes 
etc., should be least demanding in the region of the counterjet. In fact one can envisage a situation where the jet pointing away from the observer is beamed so strongly out of our direction that the this jet is rendered practically invisible. However, the scattered light produced by the counterjet might still be visible and, thus, revealing its presence.

This idea was put forth by Gilfanov et al. (1987, henceforth GSC87) in a study of the scattered radiation originating from a point source, which was modeled to vary over time and to radiate non isotropically. It was shown that a highly directional compact source can produce extended jet-like structures in the surface brightness distribution of the scattered radiation. Furthermore, GSC87 demonstrated that in addition to a temporal variability a rotation as well as a precession of the directional radiation diagram of the central point source give rise to a large amount of morphological variety of the diffuse sources of radiation.

In the context of similar studies Wise \& Sarazin (1992) and Sarazin \& Wise (1993) point out that observations of electron scattered profiles in cluster radio sources would provide a test of theories, which seek to unify disparate types of AGN, viz. the hypothesis that Fanaroff-Riley Class I (FR I) radio galaxies are the parent population of BL Lac objects. The radiation beam produced by a FR I radio source, which is pointing towards us in a BL Lac object, should be observable by means of the scattered radiation it produces.

For the purposes of the above-cited studies it was appropriate to model the radiation field produced by a relativistic jet as two oppositely directed radiation cones of constant intensity with a half-opening angle $\sim 1 / \gamma$, where $\gamma$ is the bulk Lorentz factor of the jet flow. Although this approximation certainly is valid on scales much larger than the jet itself (as will be confirmed by the results of this paper), the effects caused by the exact shape of the beamed radiation pattern as well as by the motion of the radiation source itself should become noticeable as one "approaches" the jet, i.e. as one is interested in the behaviour of the scattered radiation in the vicinity of the jet. It is, therefore, one object of this paper to study how well said approximation works on length scales which are comparable to the size of the jet itself.

This paper is structured as follows. In Sect. 2 we describe our model and the accompanying equations. We apply our model to two scenarios in Sect. 3 and present the numerically obtained solutions thereof. We use these results in Sect. 4 to try to derive constraints on the inclination angle and the bulk Lorentz factor for the observationally well studied jet of M 87. Section 5 closes with a summary of our results.

\section{The model}

Radio observations have revealed the complex structure of extragalactic jets (e.g. Bridle \& Perley 1984). Bright patches moving with apparent superluminal motion often alternate with fainter regions along a given jet. The origin of these bright patches, the so-called knots, is still not fully understood. The most popular model at present, the internal shock model (Rees 1978), regards these knots as jet plasma excited by shocks moving along the jet. Particles are accelerated by these shocks and emit radiation by the synchrotron process as they gyrate along the magnetic field of the jet. According to this model, the velocity of a knot does not necessarily equal the actual velocity of the underlying fluid flow, should, however, represent an upper limit thereof.

In another, somewhat less popular model these knots are interpreted as magnetised clouds of relativistic electrons, socalled plasmoids or blobs, being ejected out of the centre of an AGN by a cataclysmic event along the jet axes (e.g. van der Laan 1963). The velocity of these blobs by definition corresponds to the velocity of the jet flow.

Irrespective of which of the above models is "correct", in the following sections it is our aim to construct a simple albeit realistic model of the radiation field produced by a two-sided, symmetric relativistic jet. According to our model the jet spans an inclination angle towards the line of sight $\theta$, exhibits a bulk Lorentz factor $\gamma$ and radiates with an intrinsic luminosity $L_{\text {jet }}$. We imagine the jet to be made up of several point sources moving along the jet axis, which we will henceforth call "blobs".

We choose our system of coordinates as shown in Fig. 1. The electron number density $n_{\mathrm{e}}(r)$ is distributed in a spherically symmetrical way about the centre of the AGN. The $x$ and $y$-axis span the plane of the sky, with the $x$-axis being the projection of the jet axis onto the plane of the sky. The $z$-axis is pointing towards the observer. The jet is inclined with respect to the line of sight by an angle $\theta$ and the counterjet by an angle $\pi-\theta$, respectively.

At a time $t=0$ a first pair of blobs is created inside the nucleus of the AGN. Information about this event reaches the observer, located at a distance $d$ along the $z$-axis, after a time $t=d / c$, where $c$ is the speed of light. The blobs are assumed to move ballistically with a velocity $v=\beta c$ along the jet and the counterjet, respectively, wherein $\beta$ is given by $\beta=\sqrt{1-1 / \gamma^{2}}$. The motion of a blob can be described in the chosen coordinate system as

$\boldsymbol{r}_{\text {blob }}(t)=\boldsymbol{r}_{\mathrm{o}}+\boldsymbol{v} t=\left(x_{\mathrm{o}}+v_{x} t, 0, z_{\mathrm{o}}+v_{z} t\right)$,

with the projections of the blob velocity onto the coordinate axes $v_{x}=v \sin \theta$ and $v_{z}=v \cos \theta$, respectively. For a single pair of blobs $x_{\mathrm{o}}$ and $z_{\mathrm{o}}$ could be omitted. However, these quantities become necessary once one wishes to keep track of several pairs of blobs over time as described in the later sections of this paper.

The apparent velocity $v_{\text {app }}$ of the blobs in the plane of the sky, as seen by the distant observer, is given by the well known formula for superluminal motion (Rees 1966, 1967)

$v_{\text {app }}=\frac{v \sin \theta}{1 \mp \beta \cos \theta}$.

After the blobs have traveled a physical distance $l_{\text {jet }}$ corresponding to a projected distance $x_{\text {jet }}=l_{\text {jet }} \sin \theta$ along the jet and counterjet they cease radiating. The lifetime of these blobs is therefore given by $\tau=l_{\text {jet }} / v$. Due to time travel effects the apparent lifetime $\tau_{\text {app }}$ of the approaching blob differs from the one of the receding blob, which can be expressed as (e.g. Ryle \& Longair 1967)

$\tau_{\text {app }}=\tau(1 \mp \beta \cos \theta)$. 


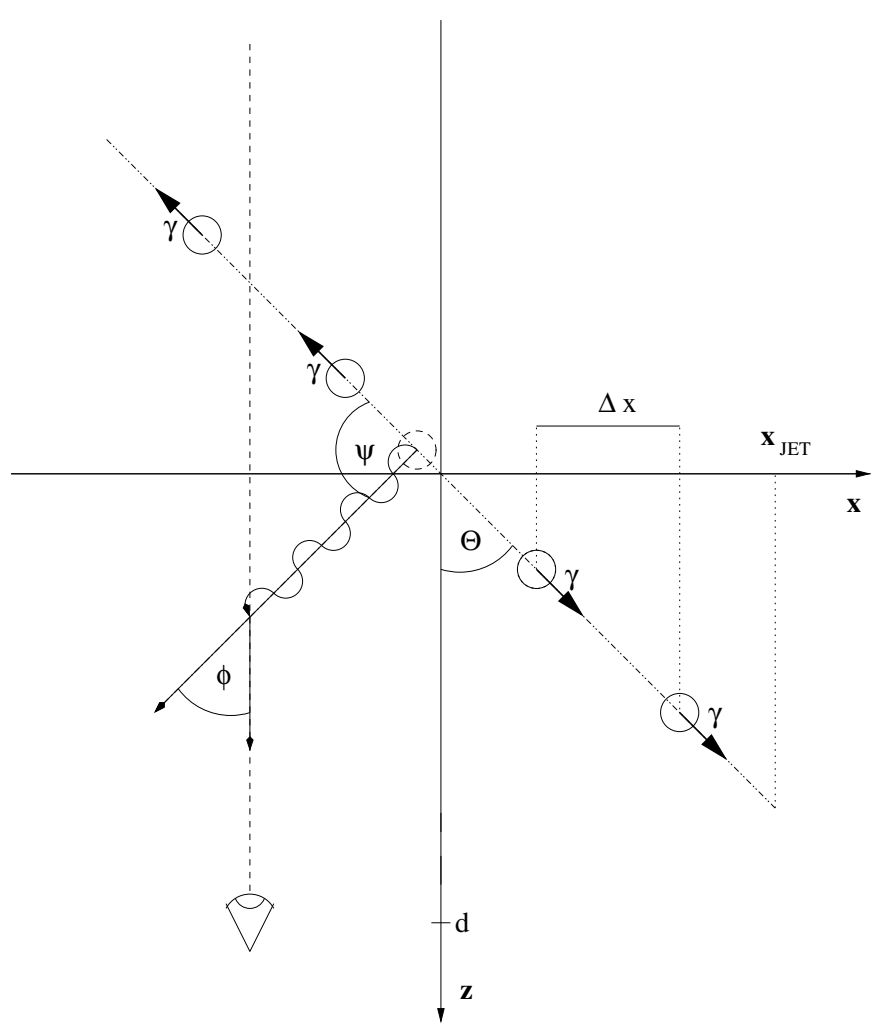

Fig. 1. A sketch of the scenario studied in this paper. An AGN situated at the origin produces a two-sided, symmetric jet lying in the $(x, z)$ plane. The jet's radiation field is modeled by a chain of "blobs" ejected by the AGN at a constant rate along directions spanning an angle of $\theta$ and $\pi-\theta$ with the line of sight of the observer, who is located at a distance $d$ along the $z$-axis. These blobs (in this case two blobs on each side) travel with a relativistic velocity $\beta c$ corresponding to a Lorentz factor $\gamma$ along the respective jet axes. The blobs are separated by a projected physical distance $\Delta x$. The actual observed apparent distance between the blobs $\Delta x_{\text {app }}$ differs for the two sides of the jet. After a blob has traveled a projected distance of $x_{\text {jet }}$ away from the AGN, according to our model the blob ceases radiating. An exemplary photon (curly line), which was produced by one of the blobs at a time when the blob was closer to the nucleus (position marked by a dashed circle), is emitted along a direction making an angle $\psi$ with the direction of the blob motion along the counterjet. Further along its path the photon is scattered through an angle $\phi$ into the direction of the distant observer and received by him at a distance $d$.

The lifetime in the reference frame of a blob would introduce an additional factor of $\gamma$ because of the transversal Doppler effect.

After a period of time $T$ a second pair of blobs is emitted in our model and so forth. If the AGN keeps producing blobs at such a constant rate, the spatial separation between blobs will remain constant. The apparent distance between two successive blobs $\Delta x_{\text {app }}$ is simply the product of their apparent velocity and the period of the blob ejection cycle, viz.

$\Delta x_{\text {app }}=v_{\text {app }} T=\frac{\Delta x}{1 \mp \beta \cos \theta}$.

Obviously, the number of blobs per unit length of jet scales inversely proportional to this quantity. Since we are assuming the jet and the counterjet to be of equal lengths, this quantity is also proportional to the number of apparently active blobs at a given moment in time.

In the following sections the above-mentioned quantities are required in order to compute the apparent luminosity of the jet and the counterjet. The total intrinsic jet luminosity is just the sum of the individual blob luminosities of existing blobs. In our model we try to approach the limit of the radiation field produced by a continuous jet by increasing the number of blobs per unit length of the jet while keeping the assumed luminosity of the jet constant.

For optical thin conditions, which is a valid approximation in the present case, the scattered surface brightness at coordinates $x, y$ on the plane of the sky and at a time of observation $t$ is given by the integral over the emissivity due to scattering along the corresponding line of sight

$I_{v}^{\mathrm{sc}}(x, y, t)=\int_{z_{\min }}^{z_{\max }} j_{v}^{\mathrm{sc}}(\boldsymbol{r}, t) \mathrm{d} z$,

where $\boldsymbol{r}$ is the radius vector of the point of scattering and $z_{\min }$ and $z_{\max }$ are the boundaries of integration, which as will be described in more detail further below themselves depend upon the position on the plane of the sky and the time of observation, i.e. $x, y$ and $t$.

In the optical thin approximation the emissivity due to scattering can be expressed as

$j_{v}^{\mathrm{sc}}(\boldsymbol{r}, t)=n_{\mathrm{e}}(\boldsymbol{r}) \sum_{\text {blobs }} \frac{P_{v}\left(\boldsymbol{n}, t^{\prime}\right)}{\left|\boldsymbol{r}-\boldsymbol{r}_{\mathrm{blob}}\left(t^{\prime}\right)\right|^{2}}\left(\frac{\mathrm{d} \sigma}{\mathrm{d} \Omega}\right)$,

where $n_{\mathrm{e}}(\boldsymbol{r})$ is the electron number density at the position $\boldsymbol{r}$, $P_{\nu}\left(\boldsymbol{n}, t^{\prime}\right)$ the spectral power per unit solid angle emitted by a blob at a "retarded time" $t^{\prime}$ at a position $\boldsymbol{r}_{\text {blob }}\left(t^{\prime}\right)$ into a direction defined by the unit vector $\boldsymbol{n}$ and $(\mathrm{d} \sigma / \mathrm{d} \Omega)$ the differential scattering cross section. The unit vector pointing from the retarded position of the blob towards the point of scattering is given by

$\boldsymbol{n}=\frac{\boldsymbol{r}-\boldsymbol{r}_{\mathrm{blob}}\left(t^{\prime}\right)}{\left|\boldsymbol{r}-\boldsymbol{r}_{\mathrm{blob}}\left(t^{\prime}\right)\right|}$

In order to evaluate the scattered surface brightness at a time of observation $t$, the corresponding retarded time $t^{\prime}$ has to be computed. The received photon must have traveled from its point of emission, i.e. the position of the blob at a time $t^{\prime}$, via the point of scattering to the distant observer. The relation between the time of observation $t$ and the retarded time $t^{\prime}$, therefore, is given by the following expression

$c\left(t-t^{\prime}\right)=d-z+\left|\boldsymbol{r}-\boldsymbol{r}_{\text {blob }}\left(t^{\prime}\right)\right|$.

Solving this implicit equation for $t^{\prime}$ yields

$t^{\prime}=\gamma^{2} \frac{A-\sqrt{A^{2}-c^{2} B / \gamma^{2}}}{c^{2}}$,

with

$A=c^{2} t-v_{x}\left(x-x_{\mathrm{o}}\right)-v_{z}\left(z-z_{\mathrm{o}}\right)-c(d-z)$

and

$B=(c t-(d-z))^{2}-\left(\left(x-x_{\mathrm{o}}\right)^{2}+y^{2}+\left(z-z_{\mathrm{o}}\right)^{2}\right)$. 
Equation (9) enables one to compute the position of the blob at a time $t^{\prime}$ as a function of the time of observation $t$ and the point of scattering with coordinates $x, y$ and $z$.

We assume a blob to emit synchrotron radiation with a spectral index $\alpha$ (in the following sections we will adopt a value of $\alpha=0.5$, which is commonly observed in the spectra of radio jets). Thus, the spectral luminosity is given by

$L_{v}=L_{v}^{\mathrm{o}}\left(\frac{v_{\mathrm{o}}}{v}\right)^{\alpha}$.

Each blob radiates at an intrinsic rate of $L_{v}^{o}=4 \pi P_{v}^{o}$. We assume the intrinsic luminosity of a blob to remain constant over its lifetime. The relation between the spectral luminosity per unit solid angle in the observer's frame, i.e. the rest frame of the scattering electron or equivalently the rest frame of the distant observer, and in the rest frame of a blob is given by (e.g. Rybicki \& Lightman 1979)

$P_{\nu}(\boldsymbol{n})=\delta^{3+\alpha} P_{v}^{\mathrm{o}}=\left(\frac{1}{\gamma(1-\beta \cos \psi)}\right)^{3+\alpha} P_{v}^{\mathrm{o}}$,

where $\delta$ is the Doppler or beaming factor.

The angle between the direction of motion of the blob and the line connecting the retarded position of the blob, i.e. the position of the blob at a time $t^{\prime}$, and the point of scattering $\psi$ can be computed as follows. The cosine of this angle is given by the scalar product of $\boldsymbol{n}$ and the unit vector along the jet axis given by $\boldsymbol{e}_{\text {jet }}=(\sin \theta, 0, \cos \theta)$. One thus obtains

$\cos \psi=\frac{\sin \theta\left(x-x_{\text {blob }}\right)+\cos \theta\left(z-z_{\text {blob }}\right)}{\sqrt{\left(x-x_{\text {blob }}\right)^{2}+y^{2}+\left(z-z_{\text {blob }}\right)^{2}}}$,

where $x_{\text {blob }}$ and $z_{\text {blob }}$ are computed according to Eq. (1).

Since we are considering photons produced by the synchrotron process and since the scattering electrons have a temperature of several keVs, it is appropriate to use the differential cross section for Thomson scattering, i.e.

$\left(\frac{\mathrm{d} \sigma}{\mathrm{d} \Omega}\right)=\frac{3 \sigma_{\mathrm{T}}}{16 \pi}\left(1+\cos ^{2} \phi\right)$,

where $\sigma_{\mathrm{T}}$ is the Thomson cross section.

The angle between the line connecting the retarded blob position and the line of sight can be computed analogously to the angle $\psi$. The relation $\cos \phi=\boldsymbol{e}_{z} \cdot \boldsymbol{n}$, where $\boldsymbol{e}_{z}$ denotes the unit vector along the $z$-axis, yields

$\cos \phi=\frac{z-z_{\text {blob }}}{\sqrt{\left(x-x_{\text {blob }}\right)^{2}+y^{2}+\left(z-z_{\text {blob }}\right)^{2}}}$.

The density profile of the hot electron gas surrounding an AGN located inside a central galaxy of a cluster can be derived by means of the observed X-ray surface brightness profile produced by this gas. Since the computations in our model are generally performed numerically, any density distribution could be used in principle. However, for the sake of simplicity we are assuming for the time being a density distribution according to the widely used beta profile (Cavaliere \& Fusco-Fermiano 1976)

$n_{\mathrm{e}}(r)=\frac{n_{\mathrm{e}}^{\mathrm{o}}}{\left(1+\left(r / r_{\mathrm{c}}\right)^{2}\right)^{\frac{3}{2} \beta_{\mathrm{c}}}}$, which is fully parametrised by the normalisation of the electron number density $n_{\mathrm{e}}^{\mathrm{o}}$, the core radius $r_{\mathrm{c}}$ and the $\beta_{\mathrm{c}}$ parameter. The application of our results to the jet of M 87 in a later section, however, will use a recently determined density profile for this object.

Finally the integration boundaries $z_{\min }$ and $z_{\max }$ of the integral occurring in Eq. (5) have to be computed. These quantities are functions of the position on the plane of the sky $x, y$ and the time of observation $t$ and can be considered as two surfaces evolving with time. These surfaces are defined as the loci of scattering sites giving a fixed time delay for photons traveling from their point of emission to the distant observer, which divide space into three distinct regions: one region that scatters photons originating from the blob during its existence and two regions that cannot scatter any photons since either the source has not turned on yet or has already switched off.

For a stationary source of radiation the shape of these surfaces is that of a paraboloid ${ }^{1}$ with the radiation source at the apex, which can be described e.g. by the following formula (e.g. Sunyaev \& Churazov 1998)

$z=\frac{1}{2}\left(\frac{x^{2}+y^{2}}{c t}-c^{2}(t-d / c)^{2}\right)$.

In the case of a moving radiation source $z_{\min }$ and $z_{\max }$ can be computed by setting Eq. (9) equal to $t_{\min }^{\prime}$ and $t_{\max }^{\prime}$, respectively, where $t_{\min }^{\prime}$ is the time the blob started radiating and $t_{\max }^{\prime}$ the time the blob stopped radiating. Solving this equation for $z_{\text {min }}$ and $z_{\max }$, respectively, it turns out that $z_{\min }$ also can be described by Eq. (16). This, however, is actually not surprising, because our model was set up in a way that a blob is created in the nucleus of the AGN corresponding to our origin of coordinates.

Concerning $z_{\max }$ the result is just a generalisation of Eq. (16) describing the loci of equal travel times from a stationary source located at the origin to the observer, i.e.

$$
\begin{aligned}
z_{\max }= & \frac{1}{2}\left(\frac{\left(x-x_{\mathrm{blob}}\left(t_{\max }^{\prime}\right)\right)^{2}+y^{2}+z_{\mathrm{blob}}\left(t_{\max }^{\prime}\right)^{2}}{c t}\right. \\
& \left.-c^{2}\left(t_{\max }^{\prime}-(t-d / c)\right)^{2}\right) .
\end{aligned}
$$

This equation describes a similar paraboloid as Eq. (16) except that its apex corresponds to the point in space where the blob ceases radiating.

With the above equations at hand the remaining problem turns out to be a function of the variables $x, y$, and $t$, the free parameters being the intrinsic spectral luminosity of the blob or jet $L_{v}^{\mathrm{o}}$, the bulk Lorentz factor $\gamma$, the inclination angle $\theta$, the parameters of the electron density distribution, i.e. $n_{\mathrm{e}}^{\mathrm{o}}, \beta_{\mathrm{c}}$ and $r_{\mathrm{c}}$, and the length of the jet $l_{\text {jet }}$, which together with a given Lorentz factor corresponds to a given lifetime of a blob.

\footnotetext{
${ }^{1}$ It should be noted that the paraboloid is an approximation to the more general case of an ellipsoid, which is valid for the case of the source of radiation and the scattering site being at large distances from the observer. The more general case has been applied e.g. in a study of the scattering of radiation produced by the supermassive black hole at the centre of the Milky Way by giant molecular clouds located in the Galactic molecular ring (Cramphorn \& Sunyaev 2002).
} 


\section{Results}

Using Eq. (5) one is now in a position to compute the surface brightness of the diffuse scattered emission for a given scenario as a function of the position on the plane of the sky and the time of observation. In the following sections we will use the brightness temperature more commonly used in radio astronomy to express our results, i.e.

$T_{\mathrm{b}}^{\mathrm{sc}}=\frac{\lambda^{2}}{2 k_{\mathrm{B}}} I_{v}^{\mathrm{sc}}$,

where $k_{\mathrm{B}}$ is Boltzmann's constant. A wavelength of observation of $6 \mathrm{~cm}$ is assumed corresponding to a frequency of about $5 \mathrm{GHz}$.

\subsection{Single ejection}

A first scenario to apply our model to is the single ejection of a pair of blobs. In order to compare the results of the present section with the results of later sections we have chosen the following set of parameters as a reference model which may be representative of an "average" jet originating in the nucleus of the central galaxy of an "average" cluster of galaxies: $\gamma=5, \theta=30^{\circ}, L_{v}^{\mathrm{o}}=10^{32} \mathrm{erg} \mathrm{s}^{-1} \mathrm{~Hz}^{-1}, l_{\text {jet }}=2 \mathrm{kpc}$, $n_{\mathrm{e}}^{\mathrm{o}}=10^{-1} \mathrm{~cm}^{-3}, \beta_{\mathrm{c}}=2 / 3$ and $r_{\mathrm{c}}=10 \mathrm{kpc}$. Furthermore, we place the source at a distance of $16 \mathrm{Mpc}$ from the observer. The corresponding observed flux is about $8.5 \times 10^{2} \mathrm{Jy}$. For the chosen set of parameters the blobs have a "lifetime" of about 6800 years corresponding to an apparent lifetime of the approaching blob of about 1000 and of the receding blob of about 12000 years, respectively. Using Eq. (2) the apparent velocities can be computed and turn out to be $3.23 \mathrm{c}$ (about 1000 parsec per 1000 years) for the approaching blob and $0.27 \mathrm{c}$ (about 80 parsec per 1000 years) for the receding blob, respectively. As can be taken from Eqs. (5) and (6) our results easily can be converted to a different density normalisation or a different intrinsic luminosity, since the scattered surface brightness just scales linearly with these quantities.

\subsubsection{Brightness temperature}

In Fig. 2 we are plotting contours of equal brightness temperature of the scattered radiation in the plane of the sky three hundred, one thousand, three thousand and ten thousand years after the ejection of a pair of blobs out of the nucleus of an AGN for the above chosen set of parameters. The two blobs travel a projected distance of $1 \mathrm{kpc}$ along the positive and negative $x$-axis, respectively, and then stop to radiate, wherein the blob moving to the right is approaching the observer.

In the first snapshot three hundred years after the ejection of the pair of blobs the scattered radiation due to the approaching blob is already rather extended, whereas the scattered radiation produced by the receding blob is barely visible at the centre. The scattered surface brightness stemming from the approaching blob has already reached a maximum value of more than $10^{4} \mathrm{~K}$ and the position of this maximum coincides with the apparent position of the approaching blob. This maximum is about $300 \mathrm{pc}$ distant from the nucleus. Although the distribution of the scattered surface brightness due to the approaching blob is symmetric with respect to the $x$-axis, such a symmetry is not given with respect to the direction perpendicular thereto. Rather some contour lines of the scattered radiation produced by the approaching blob adopt a more "kidney-like" shape, which becomes even more pronounced in the following snapshot seven hundred years later. We have observed this shape in our numerical computations in particular for the cases of small inclination angles and large Lorentz factors. However, the contour lines at further distances from the nucleus exhibit a spherically symmetric shape.

The second snapshot one thousand years after the ejection of the pair of blobs shows the morphology of the brightness temperature of the scattered emission at a time just before the approaching blob switches off, i.e. reaches a projected distance of $1 \mathrm{kpc}$ from the nucleus. Compared with the previous snapshot the pattern of scattered radiation produced by the approaching blob has spread out further, while the maximum brightness temperature has decreased somewhat. Close to the nucleus the scattered radiation originating from the receding blob is just starting to become more extended, but already has reached a maximum brightness temperature of almost $10^{4} \mathrm{~K}$. As can be taken from this and especially the two following snapshots the scattering pattern produced by the receding blob exhibits a more spherically symmetric shape than the scattering pattern produced by the approaching blob.

Two thousand years later, i.e. three thousand years after the ejection of the pair of blobs, the maximum of scattered radiation due to the approaching blob has already moved beyond the scales of Fig. 2, i.e. to projected distances more than $2 \mathrm{kpc}$ away from the nucleus. Only a single contour line corresponding to a brightness temperature of $1 \mathrm{~K}$ is still visible on the right side of the plot. As the approaching blob already has switched off, this "afterglow" of scattered radiation keeps on moving away from the nucleus with the same apparent velocity as the approaching blob during its existence. The maximum of this afterglow actually is about $3 \mathrm{kpc}$ distant from the nucleus and will keep on moving to even larger distances, as time progresses further, while at the same time decreasing in brightness until completely fading away. The scattered radiation of the receding blob, on the other hand, still can be seen centered at a distance of about $250 \mathrm{pc}$ to the left of the nucleus.

Finally, after ten thousand years the scattered radiation produced by the approaching blob fully has disappeared (the maximum would be located at a distance of almost $10 \mathrm{kpc}$ from the nucleus now) and only the pattern of scattered radiation produced by the receding blob can be observed. In the meantime, this pattern of scattered radiation due to the receding blob has become more extended, while traveling out to a distance of about $800 \mathrm{pc}$. It should be noted that as in the case of the approaching blob, at still later times one can observe an "afterglow" of scattered radiation produced by the receding blob even after this blob has stopped radiating. Also this pattern of scattered radiation on the side of the receding blob keeps on moving with the same apparent velocity as the receding blob itself.

As already mentioned and as can be taken from Fig. 2, the respective maxima of the scattered surface brightnesses 


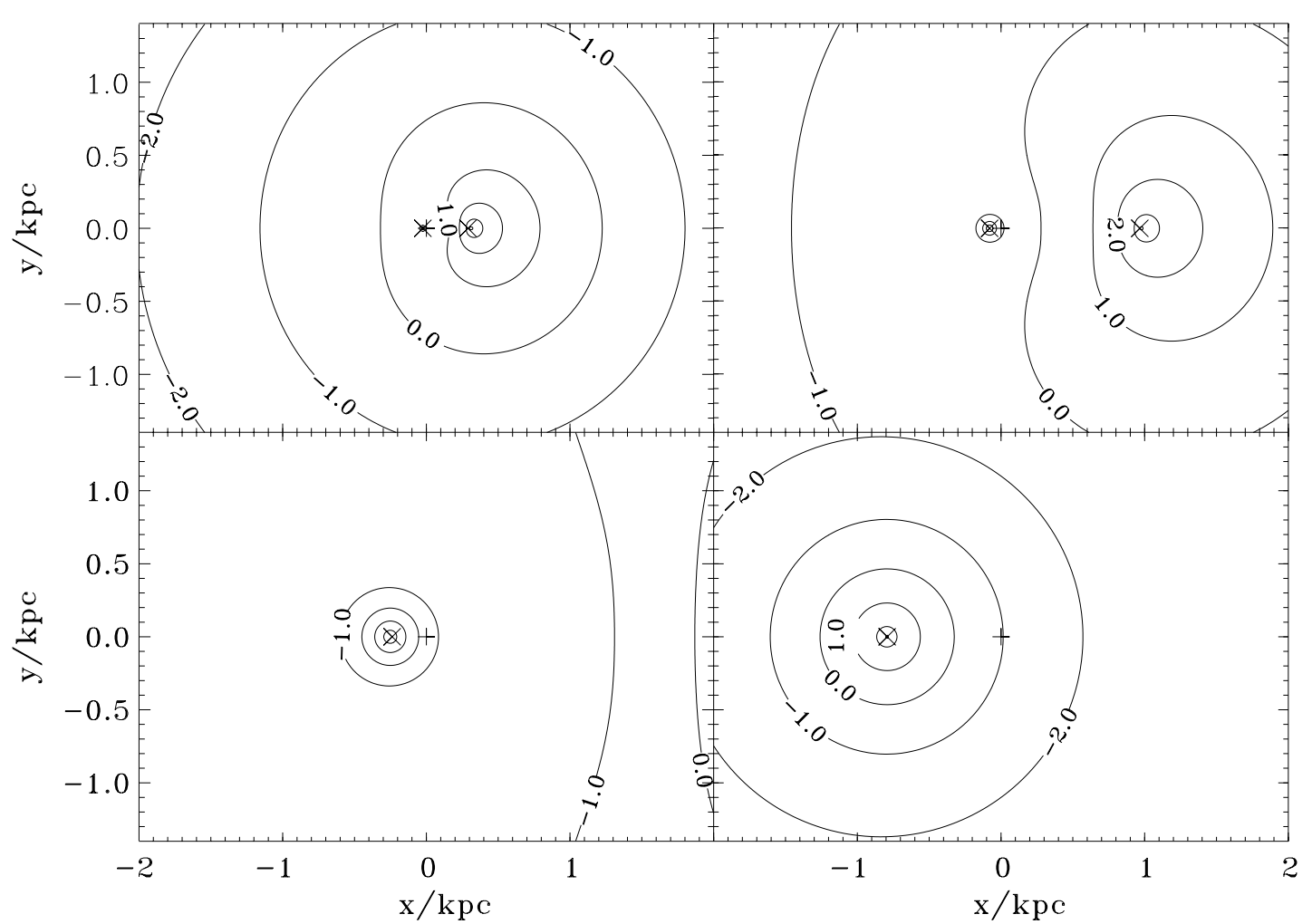

Fig. 2. Contour plots of the brightness temperature of the scattered radiation three hundred (upper left), one thousand (upper right), three thousand (lower left) and ten thousand (lower right) years after the ejection of a pair of blobs with an inclination angle of $\theta=30^{\circ}$ and a Lorentz factor of $\gamma=5$. The blob moving to the right is approaching the observer. Beyond a projected distance of $1 \mathrm{kpc}$ from the nucleus the blobs cease to radiate. The positions of the nucleus and the approaching and the receding blob are marked by a plus sign and by crosses, respectively. Logarithmic contour levels are shown down to $\log T_{\mathrm{b}}=-2$.

coincide with the respective apparent blob positions and, thus, both move with the same apparent velocity. We have verified that this holds true even for very large Lorentz factors, i.e. that the highest brightness temperature is found at the apparent position of a blob. For large Lorentz factors the scattered emission tends to become more "concentrated" around a blob.

The above results differ somewhat from the findings of GSC87, who showed that for the approximation of two oppositely directed radiation cones a burst (equivalent to our blob lifetime) produces two pronounced maxima in the brightness distribution (see Fig. 2 of GSC87), wherein the maximum on the side of the cone pointing in the direction of the observer is fainter than the one on the side of the radiation cone pointing away from the observer. Such a behaviour was not observed in our computations. Furthermore, it was shown in GSC87, that using the adopted approximation the velocities of the fainter maximum and the brighter maximum can be given by a formula solely depending on the inclination angle. Obviously, this approximation does not allow to derive any dependence on the Lorentz factor. As we have seen in our computations, however, the velocity of the scattering pattern produced by a blob is given by Eq. (2).

\subsubsection{Flux and fluence}

With respect to our model a physically somewhat more "global" quantity than the scattered surface brightness is the total scattered flux originating from a blob. In Fig. 3 we are plotting the scattered flux densities, the apparent flux densities and the fluences produced by each blob of the pair of blobs shown in Fig. 2 as a function of time up to $10^{6}$ years after the ejection. The fluxes are measured in units of Janskys, where $1 \mathrm{Jy}$ corresponds to a flux of $10^{-23} \mathrm{erg} \mathrm{cm}^{-2} \mathrm{~s}^{-1} \mathrm{~Hz}^{-1}$.

The general behaviour of the two blobs in time as exhibited in the contour plots shown in Fig. 2 and as described in the previous section is also reflected by the scattered fluxes produced by these blobs. Initially, the scattered flux due to the approaching blob dominates and increases linearly in time. About one thousand years after the ejection corresponding to the time when the approaching blob switches off, its scattered flux peaks at a maximum value of about $600 \mathrm{mJy}$. After the switch off an "afterglow" of scattered radiation remains for some time at a similar level of flux.

The lightcurve corresponding to the scattered flux due to the receding blob is similar in shape, however, peaks at a smaller maximum value and is shifted to later times. The scattered flux produced by the receding blob reaches its maximum value of about $40 \mathrm{mJy}$ about 12000 years after the ejection and remains at a similar flux level for almost $10^{5}$ years after the switch off of the receding blob.

In order to estimate a characteristic size of the distribution of the scattered radiation produced by these blobs, i.e the size of the region where the bulk of the scattered flux originates from, we have computed the flux coming from a circle of 


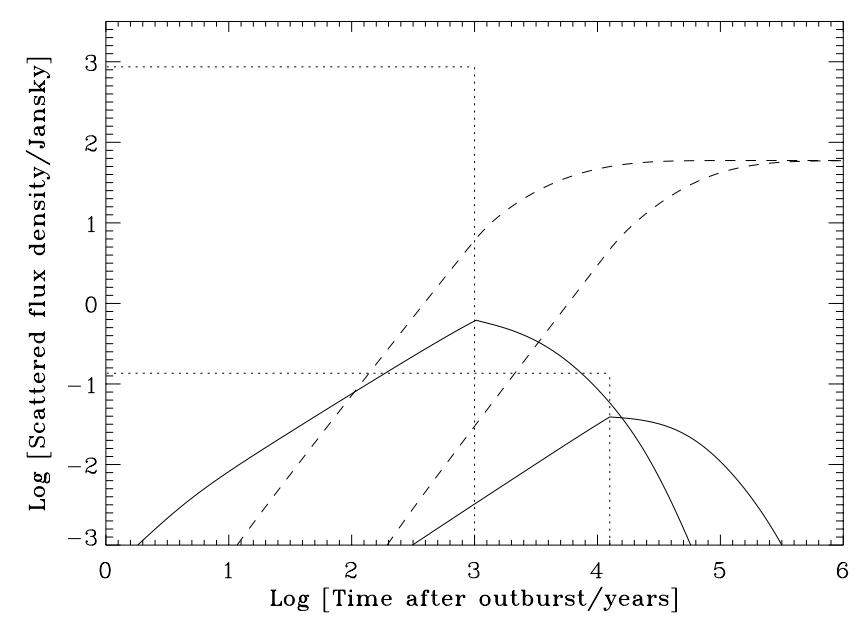

Fig. 3. Temporal evolution of the scattered flux densities (solid lines), the apparent flux densities (dotted lines) and the fluences of scattered flux (dashed lines) for the approaching and the receding blob shown in Fig. 2. As long as the approaching blob keeps on radiating the ratio of its apparent flux density to its scattered flux density is larger than three orders of magnitude. After the blobs switch off (reflected by the kinks in the lightcurves of the scattered flux and the drop off of the apparent direct flux) an "afterglow" of scattered radiation remains. The fluence of the scattered flux for both the approaching as well as for the receding blob reaches a maximum value of about $2.4 \times 10^{-16} \mathrm{erg} \mathrm{cm}^{-2} \mathrm{~Hz}^{-1}$.

radius $r$ as a function of this radius, wherein the circle is centered on the position of the blob or equivalently on the position of the maximum of the surface brightness distribution of the scattered radiation. Our results show that the size of this characteristic region grows with time, remains, however, smaller than about $1 \mathrm{kpc}$ for about $3 \times 10^{3}$ years for the approaching blob and for about $3 \times 10^{4}$ years for the receding blob.

In order to set the values for the scattered fluxes into context, we have plotted the apparent "direct" fluxes received from the approaching blob as well as from the receding blob for a comparison. Obviously these direct fluxes vanish once the blobs stop to radiate, as reflected by the drop off in flux after about 1000 years and 12000 years, respectively. While the approaching blob is active, i.e. radiating, the ratio of its apparent direct flux to its scattered flux is about 1400 . For the receding blob the ratio of the apparent direct flux to the scattered flux turns out to be a factor of about 3 .

As we have seen the approaching and the receding blob exhibit a different behaviour, when studying the surface brightness profiles produced by these blobs (Fig. 2) or their scattered flux (Fig. 3). The brightness temperature of the scattered radiation produced by the approaching blob reaches higher values and is more extended than in the case of the receding blob. Thus, the corresponding scattered flux produced by the approaching blob for a given time is larger than the scattered flux of radiation originating from the receding blob, as depicted in Fig. 3, provided that the blobs are still active. However, as also exhibited in Figs. 2 and 3 the apparent lifetime of the receding blob is correspondingly longer than the apparent lifetime of the approaching blob. It is, thus, interesting to look at the total time integrated fluxes produced by these blobs.

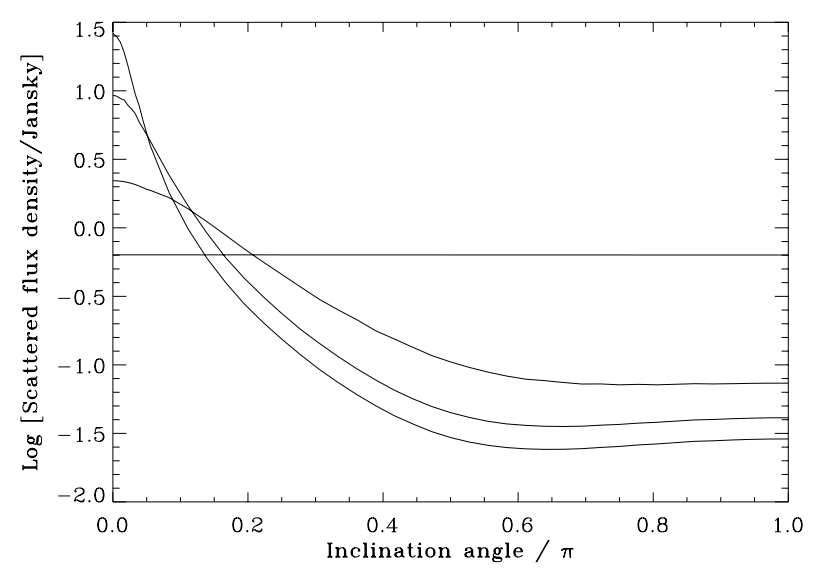

Fig. 4. Maximum of the scattered flux density produced by a single blob as a function of the inclination angle for four different Lorentz factors, viz. 1 (stationary source), 2, 5 and 10 (apart from that the same parameters have been used as before). For small inclination angles the blob moving with a Lorentz factor of 10 is producing the largest scattered flux. Note that for very large inclination angles the maximum of the scattered flux slightly starts to increase again. This effect is caused by the angular dependence of the differential cross section for Thomson scattering which "amplifies" forward as well as backward scattering compared to a scattering at a right angle.

To this end, Fig. 3 also shows the respective fluences, i.e. the time integrated fluxes, of the approaching and the receding blob. As can be taken from this figure, these fluences approach the same values as expected.

We now turn to the dependency of the maximum scattered flux produced by a single blob on the inclination angle and the Lorentz factor. Figure 4 shows this maximum scattered flux for the case of a stationary blob (which obviously shows no dependence on the inclination angle) and three blobs moving with different Lorentz factors. For small inclination angles the blob with the largest Lorentz factor, i.e. $\gamma=10$, produces the largest scattered flux, which decreases for larger inclination angles. It is interesting to note, however, that beyond a certain inclination angle the scattered flux slightly starts to increase again. We have verified that this behaviour is caused by the specific angular dependence of the Thomson scattering cross section which enhances forward and backward scattering compared with scatterings at different angles.

In order to be able to address the question, whether the scattered radiation discussed in this section might be observable at all, we have plotted in Fig. 5 the ratio of the total scattered flux produced by a blob to its apparent direct flux as a function of the inclination angle for four different Lorentz factors, viz. 1 (stationary source), 2, 5 and 10. Since for a stationary point source the scattered flux is given by $F_{\mathrm{sc}}=\tau_{\mathrm{T}} F_{\mathrm{o}}$ (Sunyaev 1982), this ratio should correspond to the optical depth for Thomson scattering. For the chosen electron density distribution the Thomson depth turns out to be about $1.85 \times 10^{-3}$.

Concerning the case of a moving blob, it can be taken from Fig. 5 that for small inclination angles the scattered flux is several orders of magnitude smaller than the direct flux, wherein this ratio decreases for larger Lorentz factors. As one goes to larger inclination angles, however, the ratio of the scattered 


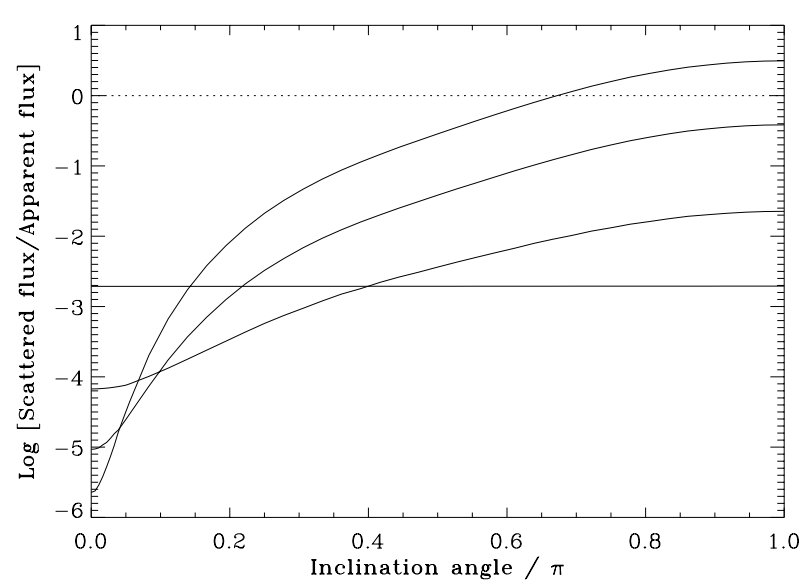

Fig. 5. Ratio of the maximum of the scattered flux density to the apparent flux for a single blob as a function of the inclination angle of the direction of motion of the blob with respect to the line of sight for four different Lorentz factors, viz. 1, 2, 5 and 10. The ratio for the stationary source is an estimate of the optical depth for Thomson scattering of the surrounding gas, which in this case is about $1.85 \times 10^{-3}$. Given the assumed electron density and the corresponding Thomson depth, the ratio of the scattered flux to the apparent flux reaches unity at an inclination angle of about $2 / 3 \pi$ for a Lorentz factor of 10 . For even larger inclination angles the scattered flux becomes larger than the apparent flux.

flux to the direct flux increases substantially. For a Lorentz factor of 10 this ratio reaches unity at an inclination angle of about $120^{\circ}$. For even larger inclination angles the flux of scattered radiation becomes larger than the flux of direct radiation given a Lorentz factor of 10. For Lorentz factors of 5 and below the ratio does not reach unity for the chosen set of parameters. However, since the scattered radiation is simply proportional to the electron density and the direct emission does not depend thereon, the ratio of these two quantities scales linearly with the assumed electron density.

\subsection{Multiple ejections}

In this section we will study a multiple blob scenario, i.e. instead of creating only a single pair of blobs a second pair of blobs is ejected after a certain period of time and so forth. If the ejection period is chosen to be short enough, the ambient scattering medium will "sense" no difference to a quasi-continuous ejection of radiating matter moving at relativistic velocities. As will become clear from the results presented in this section and as can be taken from Eqs. (5) and (6), the problem we are studying is linear in the contribution of each single blob. Thus, the scattering pattern produced by several blobs essentially is just the sum of several spatially and temporally shifted scattering patterns produced by a single blob as illustrated in Fig. 2 .

\subsubsection{Brightness temperature}

Figure 6 shows a contour plot of the brightness temperature of the scattered radiation about 20000 years after the ejection of a first pair of blobs for the multiple blob scenario. A pair of blobs is being ejected every 1000 years corresponding to

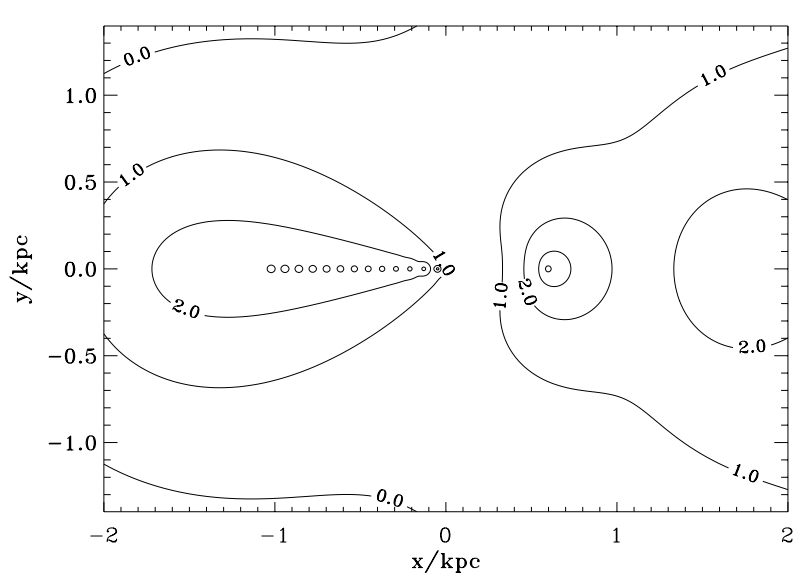

Fig. 6. Contour plot of the brightness temperature of the scattered radiation about twenty thousand years after the onset of the jet activity, i.e. the ejection of the first pair of blobs. About every 1000 years a further pair of blobs is being produced. The corresponding apparent distance between individual blobs is about $1 \mathrm{kpc}$ for the approaching blobs on the right side of the nucleus and about $80 \mathrm{pc}$ for the receding blobs on the left side of the nucleus.

an intrinsic spatial blob separation of about 300 pc. Given a Lorentz factor of 5 and and an inclination angle of $30^{\circ}$, the apparent distances between two subsequent blobs can be computed according to Eq. (4) yielding a distance of about $1 \mathrm{kpc}$ between approaching blobs and about 80 pc between receding blobs, respectively. These different apparent distances between blobs for the jet and the counterjet side are readily apparent in Fig. 6. As before, we assume the blobs to switch off after having traveled a projected distance of $1 \mathrm{kpc}$. As, furthermore, can be taken from Fig. 6, the pattern of scattered radiation on the side of the receding blobs looks much "smoother" than the one on the jet side. However, as will become clear from the following figures, by reducing the blob separation further, also the scattering pattern on the side of the approaching blobs will adopt a more or less smooth shape.

In the following it is our aim to study the behaviour exhibited in Fig. 6 for a larger number of blobs, for longer times and on larger scales. To this end, we are plotting in Fig. 7 the brightness temperature of the scattered radiation as a function of position on the plane of the sky at $10^{3}, 10^{4}, 10^{5}$ and $10^{6}$ years after the onset of the assumed jet activity. Again, the blobs are ejected with an inclination angle of $30^{\circ}$ with respect to the line of sight, move with a velocity corresponding to a Lorentz factor of 5 and stop radiating beyond a projected distance of $1 \mathrm{kpc}$. An intrinsic spatial separation of the blobs of $50 \mathrm{pc}$ has been chosen corresponding to an ejection of a pair of blobs about every 170 years. Save to the larger spatial dimensions of the chosen field of view, the contour plots shown in this figure are analogous to the contour plots shown in Figs. 2 and 6.

Over a time span of $10^{6}$ years we are observing the gradual build up of a stationary scattering pattern initially on the side of the jet and thereafter on the side of the counterjet. In particular, it can be taken from the first snapshot in Fig. 7 that one thousand years after the onset of the jet activity the scattered radiation pattern begins to develop on the side of the approaching blobs, i.e. the side of the jet. Already at these early times 


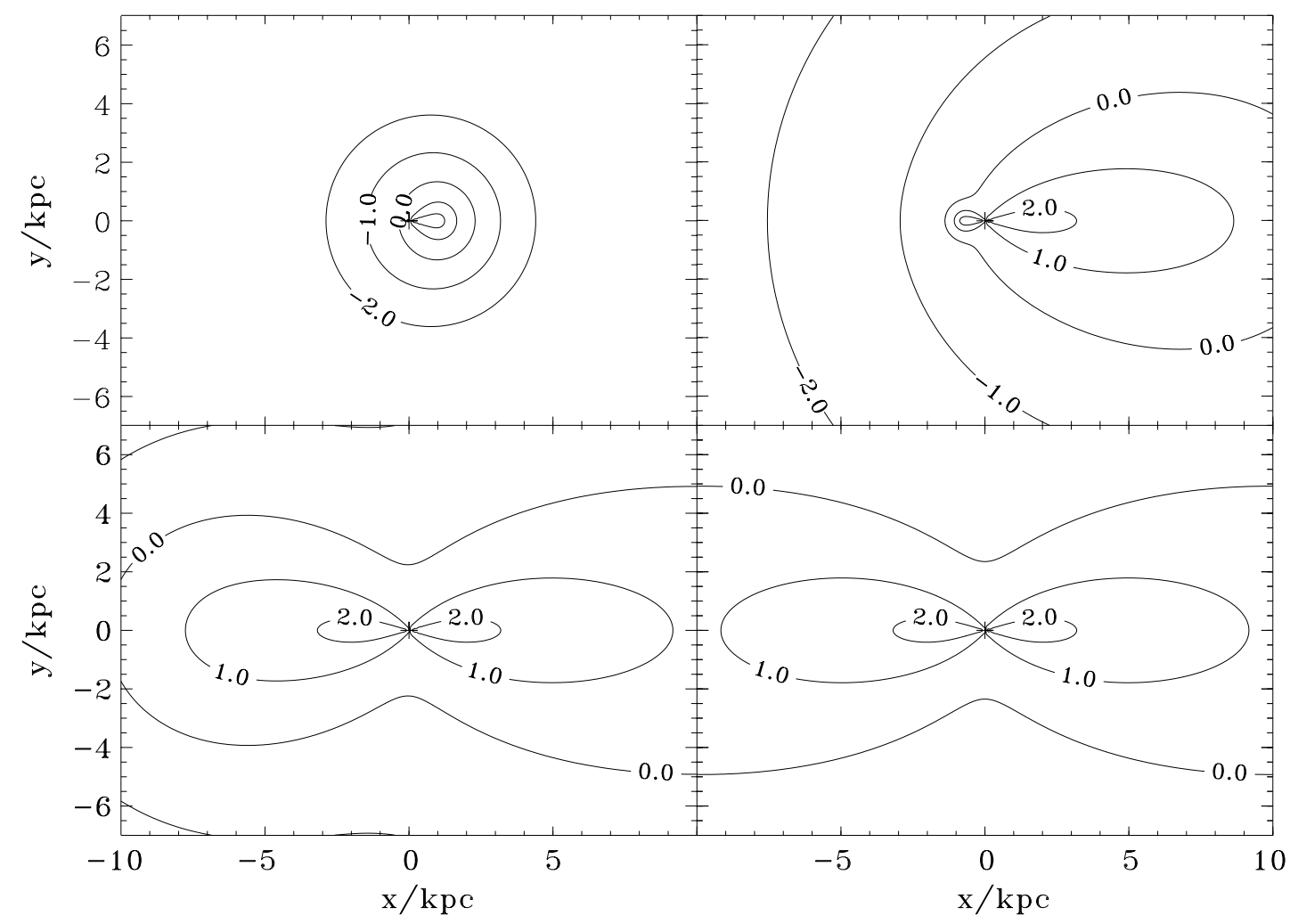

Fig. 7. Contour plots of the brightness temperature of the scattered radiation $10^{3}$ (upper left), $10^{4}$ (upper right), $10^{5}$ (lower left) and $10^{6}$ (lower right) years after the onset of the jet activity, i.e. the ejection of the first pair of blobs. About every 170 years a further pair of blobs has been ejected. The position of the nucleus is marked by a plus sign. Logarithmic contour levels are shown down to $\log T_{\mathrm{b}}=-2$.

the high brightness temperatures on the side of the approaching blobs adopt an elongated jet-like shape close to the nucleus. The contours of lower brightness temperatures at larger distances from the nucleus exhibit a more symmetric shape. On this scale one cannot discern any contribution to the scattered radiation produced by the blobs on the side of the counterjet yet.

However, 9000 years later scattered radiation produced by the receding blobs can be observed up to a distance of $1 \mathrm{kpc}$ from the nucleus exhibiting a jet-like shape similar to the one produced on the side of the jet in the earlier snapshot. The scattering pattern on the jet side, on the other hand, has in the meantime almost fully developed on the scales shown in this figure, especially in the vicinity of the nucleus. Further generations of approaching blobs will maintain this "steady state".

One hundred thousand years after the onset of the jet activity, the picture is nearly symmetric with respect to the $y$-axis, i.e. the scattering patterns on the jet and the counterjet side appear almost identical. Only for distances of about $10 \mathrm{kpc}$ to the left of the nucleus the scattered radiation on the side of the counterjet is still a bit fainter in comparison with the corresponding regions on the jet side. In other words, the scattering pattern on the side of the counterjet has not "caught up" completely with the scattering pattern on the jet side yet, which as mentioned before by this time already has reached a stationary shape.

In the last snapshot of Fig. 7 illustrating the situation $10^{6}$ years after the onset of the jet activity the whole pattern of scattered radiation has reached a symmetric, stationary shape. Differently put, if we would keep producing blobs at the adopted rate, the morphology exhibited by the scattered radiation obviously would not change. This "steady-state" scattering pattern is reminiscent of the plot presented in GSC87 (see their Fig. 1), which was obtained by a point source radiating into two oppositely directed cones. Obviously, on the scales shown in Fig. 7 such an approximation produces similar results, since the projected length of the jet and the counterjet of $1 \mathrm{kpc}$ is small compared to the dimensions of the whole system illustrated in this figure. Essentially, one can think of the final snapshot shown in Fig. 7 being made up of several "overlapping", more or less spherically symmetric patterns produced by a single blob as shown in Fig. 2.

Now that we have been producing blobs for $10^{6}$ years and the scattered surface brightness exhibits a symmetric shape, we want to study what happens when the jet switches off, i.e. when no more blobs are being ejected. Figure 8 shows the effect of such a switch off by means of contour plots of the brightness temperature of the scattered radiation one thousand, three thousand, ten thousand and thirty thousand years after the demise of the jet activity.

Initially, the brightness temperature in the vicinity of the nucleus drops on the side of the jet. As time progresses, this region of reduced brightness moves out to larger distances from the nucleus. After about ten thousand years a similar behaviour can be observed on the side of the counterjet. By the time of thirty thousand years after the demise of the jet activity the scattered emission on the side of the jet has vanished completely. However, regions on the side of the counterjet being more 


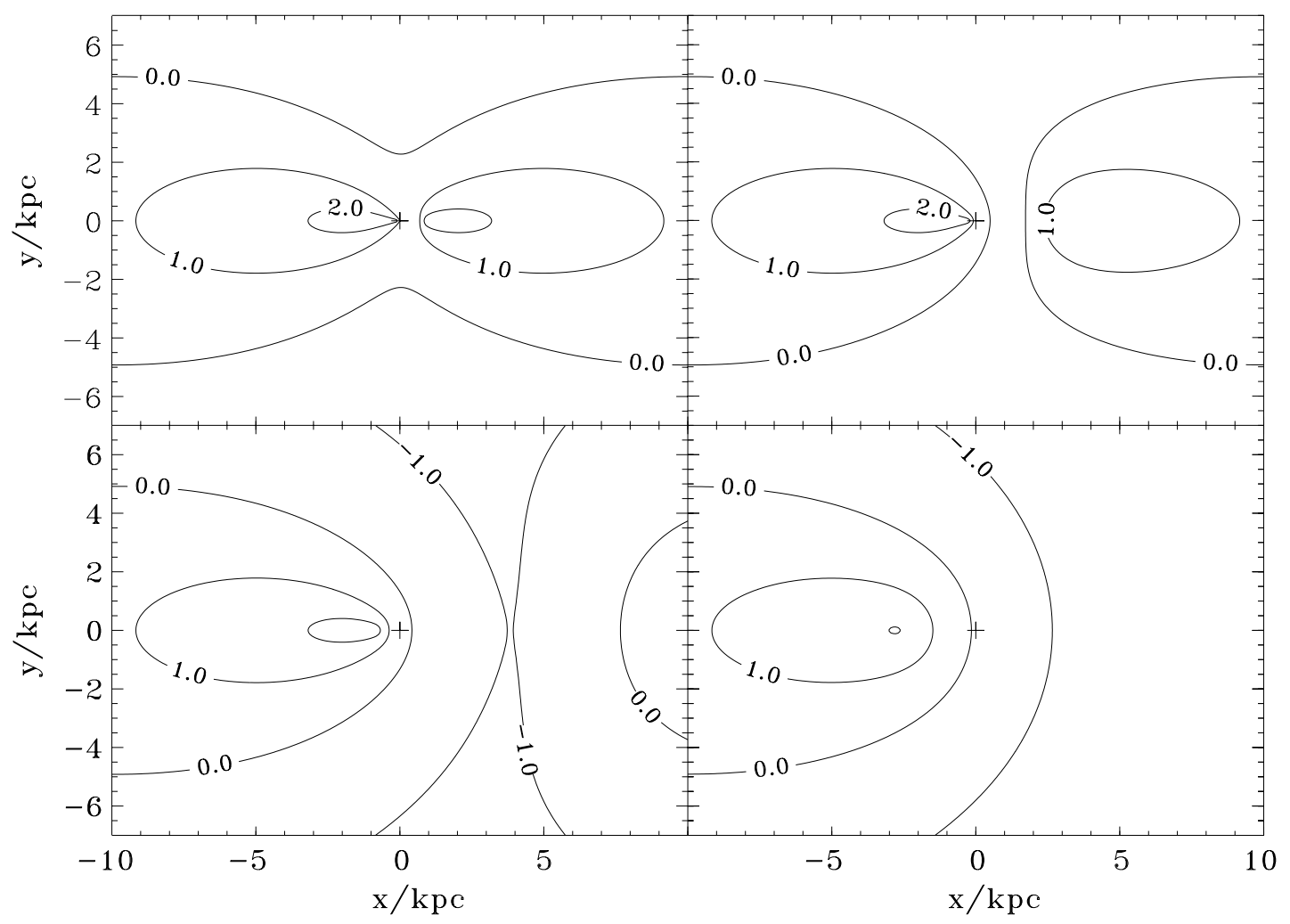

Fig. 8. Contour plots of the brightness temperature of the scattered radiation one thousand (upper left), three thousand (upper right), ten thousand (lower left) and thirty thousand (lower right) years after the demise of the jet activity, i.e. the ejection of the last pair of blobs, which lasted for $10^{6}$ years (corresponding to the snapshot shown in the lower right of Fig. 7). The position of the nucleus is marked by a plus sign. Logarithmic contour levels are shown down to $\log T_{\mathrm{b}}=-2$.

distant from the nucleus still exhibit some brightness, wherein the maximum brightness temperature is located at about $3 \mathrm{kpc}$ to the left of the nucleus. At even later times the maximum of this afterglow of scattered radiation will have traveled to greater distances from the nucleus and will have decreased further in brightness.

\subsubsection{Flux and fluence}

As in the section discussing the scenario with a single pair of blobs, we now turn to the scattered fluxes in order to study how the general behaviour exhibited in Figs. 7 and 8 is reflected by these quantities.

Figure 9 being similar to Fig. 3 shows the scattered flux, the direct flux and the fluence produced by the jet and the counterjet as a function of time after the onset of the jet activity in our multiple blob scenario. Given the assumed set of parameters, the scattered flux due to the counterjet reaches the same level as the scattered flux due to the jet after about three hundred thousand years corresponding to the stationary scattering pattern illustrated in the snapshot on the lower right of Fig. 7. However, as in the case of a single pair of blobs initially the flux due to the approaching blobs dominates over the flux of scattered radiation produced by the receding blobs. It should be noted that the lightcurve shown in Fig. 9 is essentially just the sum of the lightcurves for a single blob as shown in Fig. 3 shifted by a constant time difference.

The behaviour of the scattered fluxes after the switch off is difficult to assess from Fig. 9. We, therefore, have plotted the scattered flux and the apparent flux after the switch-off in Fig. 10 using a different time axis. As in the case of a single pair of blobs, one can observe an afterglow of diffuse scattered radiation after the apparent fluxes have died away that lasts substantially longer on the side of the counterjet. Note that in this case the switch-off of the jet is not a simple step function, but rather the sum of multiple step functions shifted in time.

We now want to study the dependence of the quantities discussed above on the inclination angle and the Lorentz factor. To this end Fig. 11 shows stationary scattering patterns for four different inclination angles, viz. $15^{\circ}, 30^{\circ}, 60^{\circ}$ and $90^{\circ}$. The other parameters have been chosen as in the previous examples. The scattering patterns in question which exhibit a symmetry with respect to the $x$ - and the $y$-axis are reached after a quasicontinuous ejection of blobs for roughly $4 \times 10^{5}, 2.5 \times 10^{5}$, $10^{5}$ and $5 \times 10^{4}$ years, respectively. These values were estimated by computing the time, when the flux inside the region shown in the snapshots of Fig. 11 reaches and remains at the same constant value on the jet side as well as on the side of the counterjet. For this reason in the case of $\theta=30^{\circ}$ the time to reach the steady state is a bit shorter than the time deduced from Fig. 9, because in that figure the total scattered flux is 


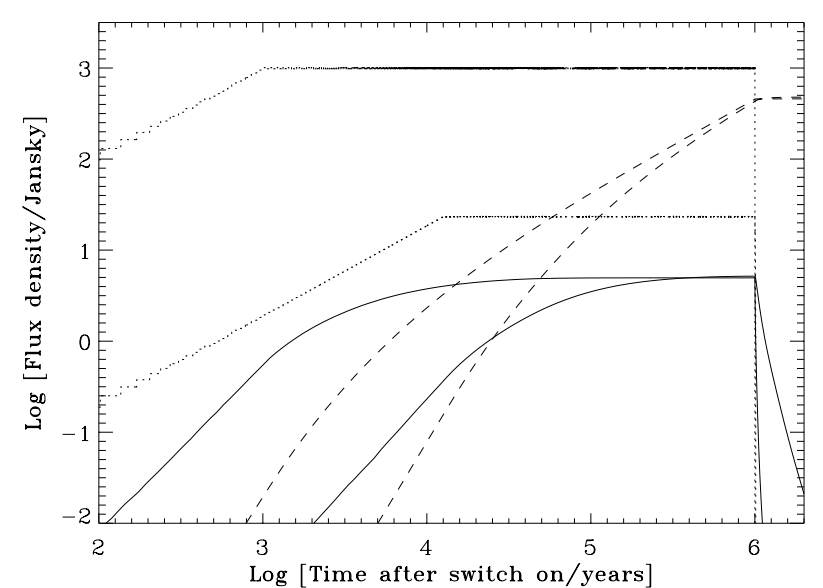

Fig. 9. Temporal evolution of the scattered flux densities (solid lines), the apparent flux densities (dotted lines) and the fluences of scattered flux (dashed lines) for the jet and the counterjet shown in Figs. 7 and 8. After a time of about $3 \times 10^{5}$ years the counterjet produces the same amount of scattered flux as the jet as long as the source is active. The fluence of the scattered flux for both the jet as well as for the counterjet reaches a maximum value of about $4.6 \times 10^{-13} \mathrm{erg} \mathrm{cm}^{-2} \mathrm{~Hz}^{-1}$.

shown, to which regions beyond the scales of the snapshots given in Fig. 11 contribute.

The time needed to reach the stationary state obviously increases with decreasing inclination angle. The reason for this behaviour is as we have seen in the previous section that for a given Lorentz factor it takes the receding blobs apparently longer to travel out to the projected distance where they stop radiating.

Concerning the overall shape of the distribution of the scattered radiation, these scattering patterns appear more "elongated" along the $x$-axis for the cases where the jet axis lies close to the plane of the sky, i.e. inclination angles of roughly $90^{\circ}$. In other words, for these cases more flux is contributing to the total scattered flux from regions located at larger distances from the nucleus. It is interesting to compare these plots with the corresponding plots for a point source radiating into two oppositely directed cones as given in GSC87 for the case of $\theta=90^{\circ}$ (see their Fig. 1) and by Wise \& Sarazin (1992) for the case of $\theta=15^{\circ}$ and also $\theta=90^{\circ}$ (see their Fig. 7, assuming an opening angle of the cone with a half angular width of $15^{\circ}$ ). When looking for instance at the respective surface brightness distributions for the case where the jet axes lie in the plane of the sky it seems that taking into account the actual blob motion has the effect of stretching out the whole scattering pattern along the negative and positive $x$-axis, respectively. Obviously, the plots provided in the earlier studies cannot provide any brightness temperature for regions lying outside the assumed radiation cones. However, save to the above mentioned difference the results inside of the radiation cones appear qualitatively similar.

The above described behaviour is also apparent when looking at the dependence of the total scattered flux on the Lorentz factor and especially on the inclination angle. Figure 12 shows the maximum of the scattered flux density produced by a jet as a function of the inclination angle for three different Lorentz

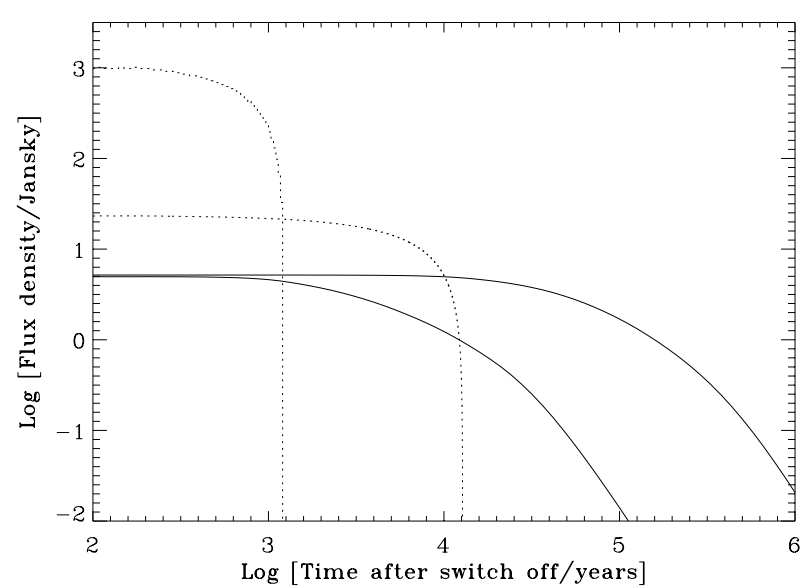

Fig. 10. Temporal evolution of the scattered flux densities (solid lines) and the apparent flux densities (dotted lines) for the jet and the counterjet shown in Fig. 8 after the demise of the jet activity, which lasted for $10^{6}$ years.

factors. As can be taken from this figure, the total scattered flux does not depend strongly on the Lorentz factor or the inclination angle.

The behaviour exhibited in Fig. 12 might at first sight seem a bit surprising, in that the dependency of the total scattered flux on the inclination angle and the Lorentz factor turns out to be so small. However, since essentially the same amount of energy, i.e. the same number of photons, is distributed over more or less the same volume, one would expect no "substantial" differences between the respective total scattered fluxes once the distribution of scattered radiation has settled to a stationary state. We have verified that the small dependency of the total scattered flux on the inclination angle and the Lorentz factor apparent from Fig. 12 is primarily due to the angular dependence of the Thomson scattering cross section as well as to a lesser extent to the chosen density distribution.

In Fig. 13 we are plotting the ratio of the total scattered flux to the apparent flux for our simulated jet having reached its stationary state as a function of the inclination angle with respect to the line of sight for three different Lorentz factors. The conclusions to be drawn from this figure are rather similar to the results given in Fig. 5, in that above a certain inclination angle of in this case about $140^{\circ}$ the total scattered flux can dominate the apparent direct flux in the case of a Lorentz factor of ten or larger. It should, however, be kept in mind that in computing the ratio we have used the total scattered flux, to which as was shown in the previous contour plots regions can contribute of sizes much larger than the jet itself.

For Lorentz factors equal or smaller than five the apparent direct flux dominates the total scattered flux for any inclination angle. These curves do not depend on the assumed intrinsic jet luminosity, but scale linearly with the assumed electron density. Since as we have seen in the previous figure the total scattered flux varies very little with the inclination angle, the strong dependence of the ratio given in Fig. 13 on the inclination angle essentially is due to the variation of the beaming factor on the Lorentz factor and the inclination angle. This point will be of importance in the following section where we try to derive 


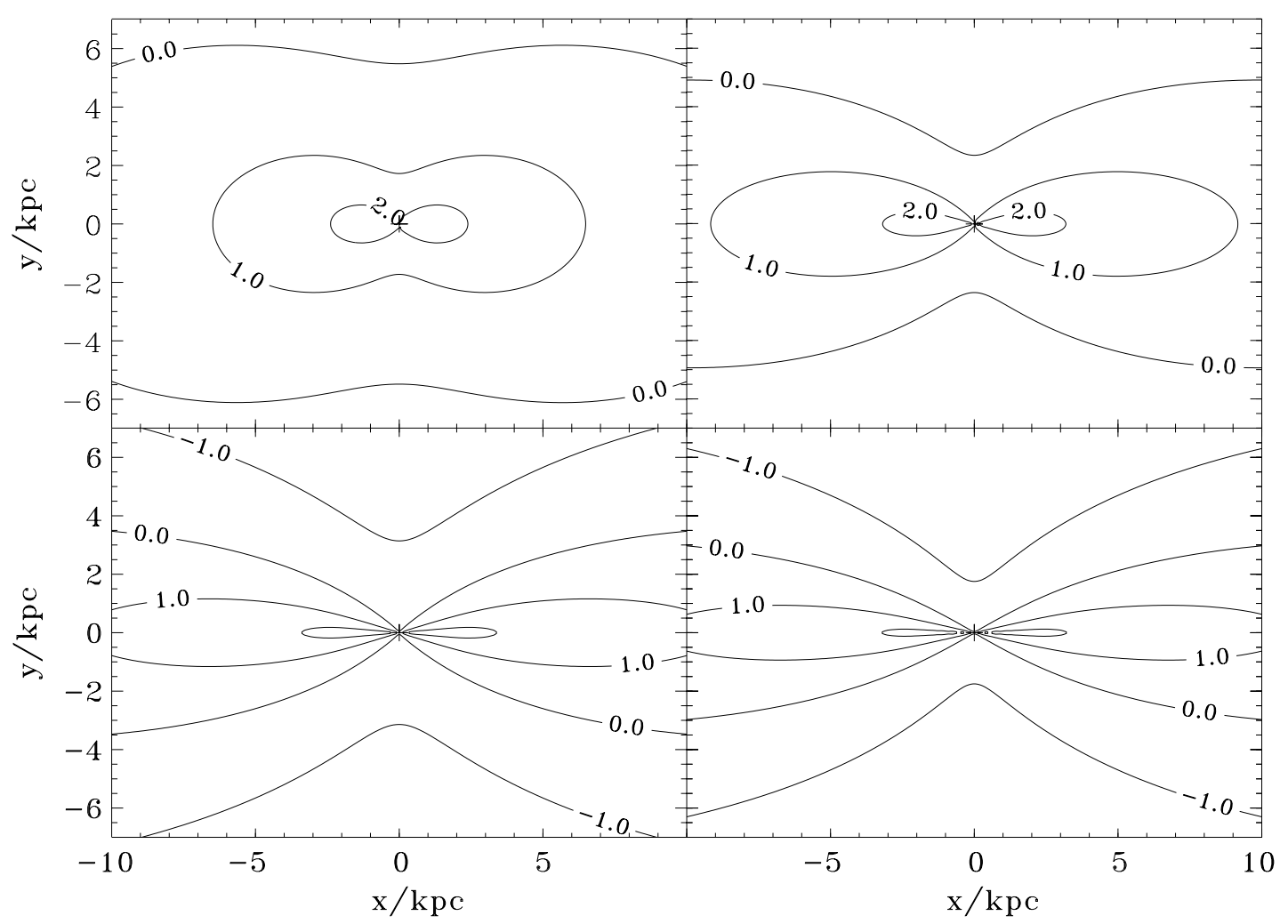

Fig. 11. Contour plots of the brightness temperature of the scattered radiation for the stationary, symmetric case for an inclination angle of $15^{\circ}$ (upper left), $30^{\circ}$ (upper right), $60^{\circ}$ (lower left) and $90^{\circ}$ (lower right). The time required to reach such a stationary state is roughly $4 \times 10^{5}$, $2.5 \times 10^{5}, 10^{5}$ and $5 \times 10^{4}$ years, respectively. The position of the nucleus is marked by a plus sign.

constraints on the parameters of the jet of M 87 based on the above described models.

\section{Application to M 87}

\subsection{Observations}

M 87 (NGC 4486) is a giant elliptical galaxy near the centre of the Virgo cluster, which is located at a distance of about $16 \mathrm{Mpc}$ (Tonry 1991; 1 arcsec corresponds to 78 pc). It contains one of the best studied synchrotron jets as it is one of the nearest examples of this phenomenon (for a general review about the jet in M 87 see e.g. Biretta 1993). M 87 is classified as a FR I type radio galaxy, meaning a low-luminosity, centrebrightened source. Its approximately $2.5 \mathrm{kpc}$ long jet exhibits a complex knotty structure and is a prominent source of radio, optical and X-ray emission (Biretta et al. 1991). The jet has a luminosity of $L_{\text {jet }} \sim 10^{43} \mathrm{erg} \mathrm{s}^{-1}$, with the brightest knots contributing a few times $10^{42} \mathrm{erg} \mathrm{s}^{-1}$. This non thermal activity is believed to ultimately be driven by a central black hole of a mass of about $3 \times 10^{9} M_{\odot}$ (e.g. Macchetto et al. 1997). Although a counterjet has not been observed directly, a feature reminiscent of a hotspot has been detected at optical wavelengths (Sparks et al. 1992).

\subsection{Constraints on the jet parameters}

Radio observations have allowed to derive several constraints on the parameters of the jet of M 87. These constraints can be plotted in the parameter space spanned by the Lorentz factor and the inclination angle in order to study which regions thereof are consistent with the observations. In the case of M 87 such a plot has been presented e.g. by Biretta et al. (1989, 1995). Based on these observations and the constraints derived therewith, the inner jet (from the nucleus to the so-called knot A) is believed to exhibit a Lorentz factor of about 3 to 5 and to be orientated at an inclination angle of about $30^{\circ}$ to $40^{\circ}$ from the line of sight (Biretta et al. 1995).

\subsubsection{Constraint based on the flux ratio}

In deriving these constraints use was made of the fact that the ratio of the jet flux to the counterjet flux

$R_{1}=\frac{F_{\mathrm{app}}^{\mathrm{jet}}}{F_{\mathrm{app}}^{\mathrm{cjet}}}=\left(\frac{1+\beta \cos \theta}{1-\beta \cos \theta}\right)^{2+\alpha}$

has to be at least larger than the observed lower limit for this quantity of 150 , wherein $F_{\text {app }}^{\mathrm{jet}}$ and $F_{\text {app }}^{\text {cjet }}$ are the apparent fluxes from the jet and the counterjet, respectively. Obviously, Eq. (19) is only valid for the luminosity of the jet and the counterjet being equal. Less conservative estimates for this ratio provided in Biretta et al. (1989) are 740 and 1600, which were obtained by using a different technique of background subtraction. These estimates were derived by measuring the flux in a $9^{\circ}$ wide wedge centered on the jet up to a distance of about 19 arcsec from the nucleus and a corresponding region located opposite of the nucleus was used for the counterjet. 


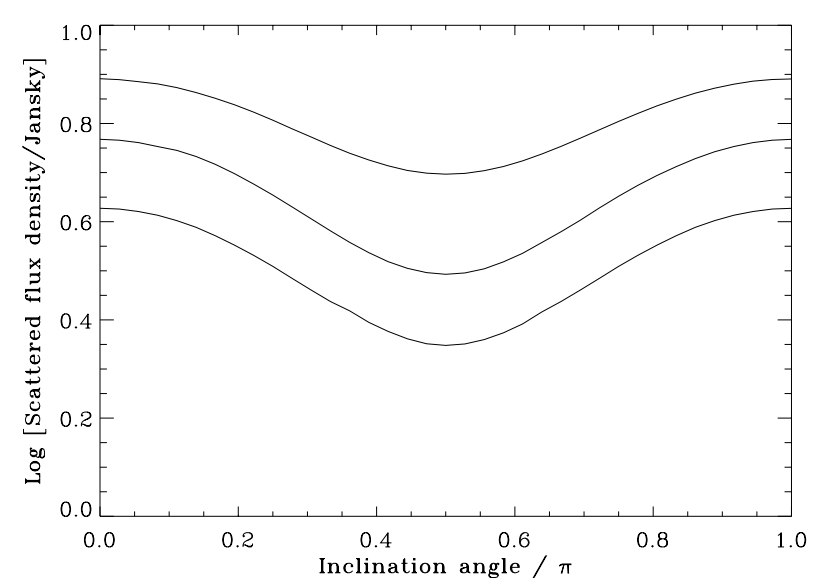

Fig. 12. Maximum of the scattered flux density produced by a jet as a function of the inclination angle for three different Lorentz factors, viz. 2, 5 and 10 (from top to bottom) for the assumed density distribution.

The above cited observational lower limits for the flux ratio also set a constraint on the scattered flux produced in the same region located on the side of the counterjet, in that also the ratio

$R_{2}=\frac{F_{\mathrm{app}}^{\mathrm{jet}}}{F_{\mathrm{sc}}^{\mathrm{cjet}}}$

has to be larger than the observational lower limits obtained by Biretta et al. (1989), where $F_{\mathrm{sc}}^{\mathrm{cjet}}$ is the scattered flux in the corresponding region located on the side of the counterjet. The model developed in the previous sections allows us to compute the ratio $R_{2}$ for a "grid" of Lorentz factors in the range from 1 to 10 and inclination angles in the range from $0^{\circ}$ to $90^{\circ}$ using the observational parameters of M 87 as follows.

Given specific values for the Lorentz factor and the inclination angle, we set up our model to reproduce the apparent flux measured on the side of the jet, which amounts to about 2.4 Jy (Biretta et al. 1989). Furthermore, we fix the observed projected length of the jet to $1.5 \mathrm{kpc}$. We model the distribution of intracluster gas around M 87 based on the results of a recent analysis of XMM observations by Matsushita et al. (2002). The electrons are distributed according to the sum of two beta-models (see Eq. (15)) with the following two sets of parameters: $n_{\mathrm{e}}^{\mathrm{o}}=0.13 \mathrm{~cm}^{-3}, \beta_{\mathrm{c}}=0.42, r_{\mathrm{c}}=1.7 \mathrm{kpc}$ and $n_{\mathrm{e}}^{\mathrm{o}}=0.011 \mathrm{~cm}^{-3}, \beta_{\mathrm{c}}=0.47, r_{\mathrm{c}}=22 \mathrm{kpc}$. We, furthermore, assume that the jet and the counterjet have been active long enough, to reach a stationary distribution of the scattered surface brightness as shown in Fig. 11. Finally, we integrate this scattered surface brightness inside a $9^{\circ}$ wide wedge having its tip centered on the nucleus and its symmetry axis coinciding with the axis of the counterjet out to a distance of about $1.5 \mathrm{kpc}$ from the nucleus yielding the scattered flux in this region.

In Fig. 14 we are plotting the two constraints $R_{1}$ and $R_{2}$ in the parameter space spanned by the Lorentz factor and the inclination angle. Obviously, the constraints derived by the methods studied in this paper and based upon the observational limit for the jet to counterjet flux ratio are less restricting than the constraints obtained by the conventional flux ratio method. Or differently put, the constraint based on $R_{1}$ already restricts the

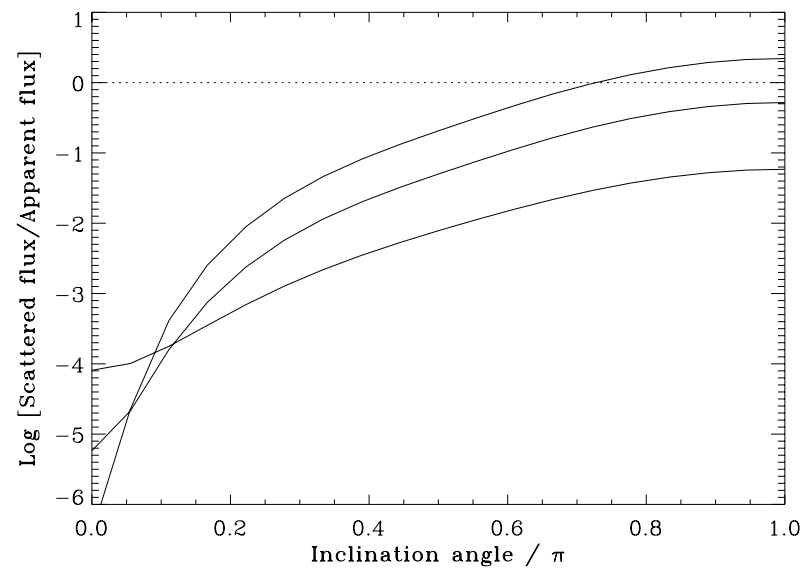

Fig. 13. Ratio of the maximum of the scattered flux density to the apparent flux for a jet as a function of the inclination angle of the jet with respect to the line of sight for three different Lorentz factors, viz. 2, 5 and 10 .

jet of M 87 to inclination angles of less than about $40^{\circ}$ and to Lorentz factors larger than about 1.5 , if one adopts the conservative value of 150 as a lower limit for $R_{1}$. For the larger ratios cited in Biretta et al. (1989) the constraints would become correspondingly more tight.

The constraint obtained by using the ratio $R_{2}$, on the other hand, allows us to exclude only a very small region of the parameter space shown in Fig. 14 corresponding to Lorentz factors larger than about 8 and inclination angles larger than about $70^{\circ}$. Even if one would adopt the larger observational limits for this ratio, in the present case the constraints based on the ratio $R_{2}$ would always be less restricting than the constraints based upon the ratio $R_{1}$, because the respective contour lines for the later ratio correspondingly would exclude more and more of the parameter space.

One of the reasons for the constraints based upon the ratio $R_{2}$ being less tight than the ones derived based on the ratio $R_{1}$ is that in computing the former ratio we, essentially, are missing a lot of scattered flux. As described above the scattered flux was computed inside a $9^{\circ}$ wide wedge out to a distance of about $1.5 \mathrm{kpc}$. However, when looking for instance at the snapshots presented in Fig. 11, one appreciates that a substantial fraction of the total scattered flux originates in regions beyond a distance of $1.5 \mathrm{kpc}$ from the nucleus and also from regions outside of the wedge in the vicinity of the nucleus. This "missing" scattered flux is also reflected in Fig. 14, where we, furthermore, have plotted the tightest constraints that could possibly be achieved by the method discussed in this section. Obviously, these "best case" constraints, in the present case however, are still less tight than the constraints based on Eq. (19) except for very large Lorentz factors of about 10 .

\subsubsection{Constraint based on the brightness temperature}

A somewhat more qualitative constraint applying the results of the previous sections of this paper can be obtained by using the observed brightness temperature in the region of the counterjet and in the vicinity of M87. To this end, we have searched in 
the literature for appropriate observations and have found two very interesting radio maps of M 87 and its environment on two different scales for the purposes of this paper.

Hines et al. (1989) present a radio map at a wavelength of $6 \mathrm{~cm}$ of the inner lobes of M 87 reaching out to a distance of about $5 \mathrm{kpc}$ from the nucleus. As can be taken from Fig. 4 of their paper the surface brightness in the region of the counterjet lies in the range between 0.38 and $1.52 \mathrm{mJy}$ per beam. It is interesting to note that this relatively dim region starts very close to the nucleus and extends out to distances of about $1.2 \mathrm{kpc}$ therefrom along the axis of the counterjet. Their observation was conducted at a wavelength of $6 \mathrm{~cm}$ and had a resolution of 0.4 arcsec.

Concerning the distribution of the surface brightness on larger scales Owen et al. (2000) recently have provided a map of the radio halo of M 87 at $90 \mathrm{~cm}$ out to a distance of about $40 \mathrm{kpc}$ from the nucleus. The radio map presented in Fig. 3 of their paper exhibits two rather interesting regions for the purposes of the present study. These regions lie on the respective extensions of the jet and the counterjet about 4 to $6 \mathrm{kpc}$ distant from the nucleus on the jet side and about 3 to $10 \mathrm{kpc}$ distant from the nucleus on the side of the counterjet. We estimate the surface brightness in these relatively dim regions "north" of the so-called feature D and "south" of the so-called feature $\mathrm{C}$ to be of the order of $20 \mathrm{mJy}$ per beam (Owen et al. 2000, in particular Fig. 3). The beam size of this observation was $7.8 \times 6.2 \operatorname{arcsec}^{2}$.

Since the measurements of the flux obtained by Biretta et al. (1989) and of the surface brightnesses in the above cited observations are given at different wavelengths, viz. 2, 6 and $90 \mathrm{~cm}$, one needs an estimate of the spectral index in order to compare these observations at different wavelengths. Harris et al. (2000) assumed spectral index values of 0.8 and 0.9 to integrate the radio spectrum between $10^{7}$ and $10^{10}$ in a study comparing extra nuclear X-ray and radio features in M87. The values for the spectral index were obtained from an unpublished spectral index map between 74 and $327 \mathrm{MHz}$ provided by N. Kassim. In a study of the radio lobes of Virgo A at $2.8 \mathrm{~cm}$ wavelength Rottmann et al. (1996) find a very steep spectrum in the outer lobes. Values for the spectral index between 1.6 and 2.8 are quoted. Adopting a spectral index of 1 , which should represent a conservative choice, we compute the brightness temperatures corresponding to the limits given by Hines et al. (1989) and Owen et al. (2000) to be 25 and $0.07 \mathrm{~K}$ at a wavelength of $2 \mathrm{~cm}$, respectively. Choosing a respective spectral index of 0.5 or 2 instead, would yield respective limits for the brightness temperature of 43 and $0.48 \mathrm{~K}$ as well as 8.3 and $0.002 \mathrm{~K}$.

Given the above derived observational limits on the surface brightness at a wavelength of $2 \mathrm{~cm}$ in two specific regions in the vicinity of M 87, we are able by using the methods presented in this paper to address the question, whether these observational limits are consistent with the scattered surface brightness to be expected from a jet producing the observed direct flux as measured by Biretta et al. (1989). Thus, in computing the respective surface brightness profiles we proceed in a similar way to the previous section while taking the beam sizes of the respective observation into account, i.e. we fix the observed apparent jet

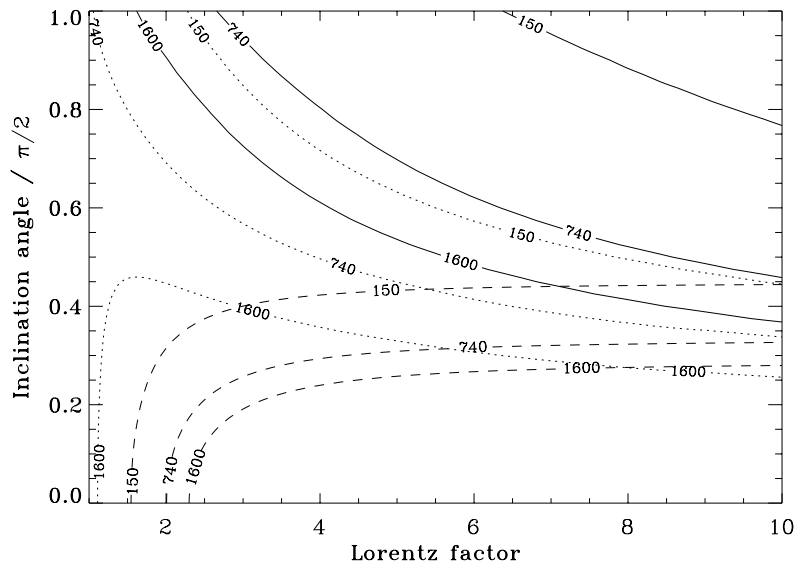

Fig. 14. Constraints on the jet of M 87 in the parameter space spanned by the Lorentz factor and the inclination angle. The solid lines mark the constraints derived by the method described in this paper based on the ratio $R_{2}$ of the apparent jet flux to the scattered flux received from a corresponding region on the side of the counterjet having the shape of a wedge with its tip centered on the nucleus. The estimated lower limits for this ratio of 150, 740 and 1600 have been taken from Biretta et al. (1989). For consistency with observations the parameters of the jet of M 87 have to lie in a region above these respective estimates, i.e. "below" the corresponding contours. The dashed lines show the conventional constraint based on the ratio $R_{1}$ of the apparent jet flux to the apparent counterjet flux. The dotted lines give the constraints according to the present paper in case it would be possible to use the total scattered flux.

flux as well as the observed projected jet length and vary the Lorentz factor and the inclination angle.

Figure 15 shows the results of these computations in that the brightness temperature of the scattered radiation is plotted as a function of the distance from the nucleus along the axis of the counterjet for three different combinations of Lorentz factor and inclination angle, viz. $\gamma=5$ and $\theta=30^{\circ}, \gamma=10$ and $\theta=30^{\circ}$ as well as $\gamma=5$ and $\theta=60^{\circ}$. Also shown are the observational upper limits taken as outlined above from Hines et al. (1989) for distances from $0.1 \mathrm{up}$ to $1.2 \mathrm{kpc}$ from the nucleus and from Owen et al. (2000) for distances from $3 \mathrm{kpc}$ up to $10 \mathrm{kpc}$ from the nucleus.

As can be taken from this figure, the presently most favoured model for the jet of $\mathrm{M} 87$ having a Lorentz factor between 3 and 5 and an inclination angle between $30^{\circ}$ and $40^{\circ}$ lies well within our constraints. However, the surface brightness profiles for the other two combinations of Lorentz factor and inclination angle, i.e. $\gamma=10$ and $\theta=30^{\circ}$ as well as $\gamma=5$ and $\theta=60^{\circ}$, do not seem to be consistent with the observations of Owen et al. (2000), in that the scattered brightness temperatures are larger than the ones observed. Obviously, for even larger Lorentz factors and/or inclination angles this inconsistency would become even more striking. Again, it should be noted, that the conclusions to be drawn from Fig. 15 are based on the assumption that the counterjet is intrinsically symmetric to the jet and that the jet has been active long enough for the scattered radiation to reach a stationary state as described in the previous sections. 


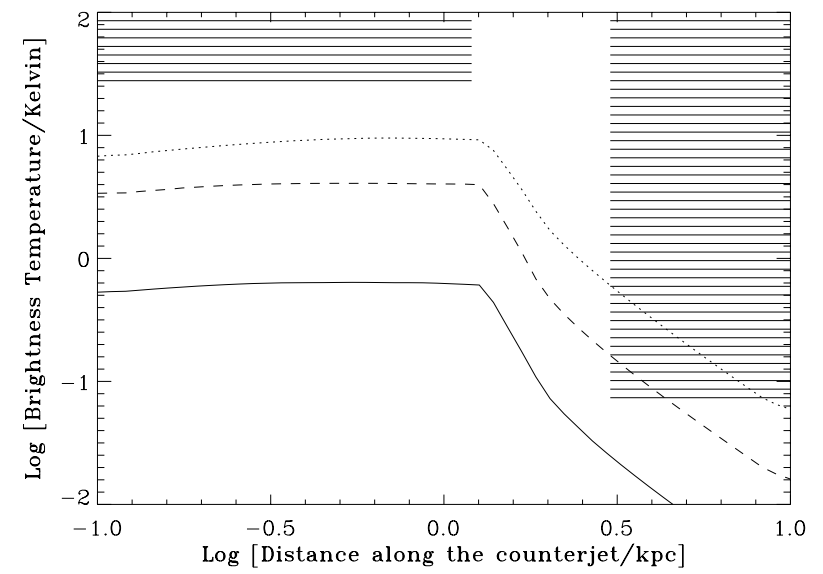

Fig. 15. Brightness temperature of the scattered radiation as a function of the distance from the nucleus along the axis of the counterjet for the following combinations of Lorentz factor and inclination angle: 5, 30 (solid curve); $10,30^{\circ}$ (dashed curve) and $5,60^{\circ}$ (dotted curve). The observational upper limits marked by rectangles filled by thick horizontal lines are taken from Hines et al. (1989) for distances of 0.1 up to $1.2 \mathrm{kpc}$ from the nucleus and from Owen et al. (2000) for distances from $3 \mathrm{kpc}$ up to $10 \mathrm{kpc}$ from the nucleus.

The surface brightness level derived from Owen et al. (2000) represents a fundamental restriction for our method which cannot be overcome by observations with a very high dynamic range, since nowhere inside the large scale lobes of the order of about $40 \mathrm{kpc}$ a lower surface brightness can be found. Furthermore, it should be noted that several features in the lobe on the side of the counterjet, i.e. the east lobe, are quite luminous (Hines et al. 1989). The total flux of these sources is about $1.5 \mathrm{Jy}$. Assuming this radiation to be isotropic the scattered flux should be about $1.5 \mathrm{mJy}$ for the assumed density distribution of M87. The regions studied in this section are located within a circle of at least $1 \mathrm{kpc}$ around this lobe. Assuming that this scattered flux is distributed over such an area in the sky, a conservative upper value for the average brightness temperature of the scattered radiation produced by the features within this eastern radio lobe turns out to be $0.05 \mathrm{~K}$ and thus should not be critical for the method studied here.

For the purposes of this paper it would be very interesting to obtain an estimate of the scattered surface brightness at higher frequencies in the region in the vicinity of M 87 we have been focusing on in this section. This is because the overall synchrotron spectrum of the jet of M 87 is characterized by a medium flat radio to optical spectral index of 0.65 , a steep cutoff at frequencies beyond a critical value of about $2 \times 10^{15} \mathrm{~Hz}$ and some flattening at frequencies below $10^{10} \mathrm{~Hz}$ (Meisenheimer et al. 1996). Given the above mentioned steep spectrum of the outer radio lobes, wherein this region is located, observations at higher frequencies, therefore, might improve the results presented here.

\section{Summary}

In this paper we have tried to assess the possibility of constraining the parameters of relativistic extragalactic jets by means of a model for the radiation field produced by such jets. Based on this model we have computed the brightness temperature of the scattered radiation as well as the scattered fluxes for several different scenarios.

In a first part of this paper we have illustrated and discussed the scattered radiation produced by a single pair of blobs being ejected along oppositely directed axes out of the nucleus of an AGN. In a second part we have studied the distribution of the scattered surface brightness produced by multiple blob pairs.

The results of these two sections have been used in a final section of this paper, where we have tried to derive independent constraints for the jet of M 87 based on the observed jet to counterjet flux ratio and based on the surface brightness in two specific regions on the side of the counterjet. We found that the presently most popular model for the jet of M 87 lies well within the derived constraints. However, a combination of a very large Lorentz factor and a large inclination angle does not seem to be consistent with the observational limits.

Lorentz factors of up to 10 have been detected in extragalactic jets. As is apparent from the foregoing results, any extragalactic jet located in a cluster with a high electron density might be a promising target for the method presented in this paper. However, motions with apparent superluminal velocities have been observed in galactic objects as well. For instance the two microquasars GRS 1915+105 and GRO J1655-40 (Mirabel \& Rodriguez 1994; Hjellming \& Rupen 1995) have an inferred bulk Lorentz factor of 2.5. However, the observations seem to suggest that the single blob scenario should be more appropriate for these cases.

Observations of Gamma-Ray Bursts (GRBs) strongly indicate and theoretical models for these objects advocate, the presence of relativistic, well collimated outflows in GRBs. The radiating matter is believed to move initially with Lorentz factors up to $10^{2}-10^{3}$. From the perspective of the present paper these objects are very interesting in that the inclination angle is most probably very well constrained, since these objects are believed to be observed very close to the line of sight. In this context it should be stressed, that in principle the method studied in this paper to constrain relativistic, well collimated outflows is not restricted to radio wavelengths. Studies in this direction have been presented by Sazonov \& Sunyaev (2003), who have demonstrated how the observed X-ray radiation from GRBs can provide information about the collimation angles of these objects.

Acknowledgements. We thank E. Churazov and M. Gilfanov for enlightening discussions.

\section{References}

Biretta, J. A., Owen, F. N., \& Cornwell, T. J. 1989, ApJ, 342, 128

Biretta, J. A., Stern, C. P., \& Harris, D. E. 1991, AJ, 101, 1632

Biretta, J. A. 1993, in Astrophysical Jets, ed. D. Burgarella, M. Livio, \& C. P. O'Dea (Cambridge University Press), 225

Biretta, J. A., Zhou, F., \& Owen, F. N. 1995, ApJ, 447, 582

Blandford, R. D., \& Rees, M. J. 1974, MNRAS, 169, 395

Bridle, A. H., \& Perley, R. A. 1984, ARA\&A, 22, 319 
Cavaliere, A., \& Fusco-Fermiano, R. 1976, A\&A, 49, 137

Cramphorn, C. K., \& Sunyaev, R. A. 2002, A\&A, 389, 252

Gilfanov, M. R., Sunyaev, R. A., \& Churazov, E. M. 1987, Sov. Astron. Lett., 13, 233 (GSC87)

Harris, D. E., Owen, F. N., Biretta, J. A., \& Junor, W. 2000 [arXiv: astro-ph/0002464]

Hines, D. C., Owen, F. N., \& Eilek, J. A. 1989, ApJ, 347, 713

Hjellming, R. B., \& Rupen, M. P. 1995, Nature, 375, 464

Macchetto, F., Marconi, A., Axon, D. J., et al. 1997, ApJ, 489, 579

Matsushita, K., Belsole, E., Finoguenov, A., \& Böhringer, H. 2002, A\&A, 386, 77

Meisenheimer, K., Röser, H.-J., \& Schlötelburg, M. 1996, A\&A, 307, 61

Mirabel, I. F., \& Rodriguez, L. F. 1994, Nature, 371, 46

Owen, F. N., Eilek, J. A., \& Kassim, N. E. 2000, ApJ, 543, 611

Rees, M. J. 1966, Nature, 211, 468

Rees, M. J. 1967, MNRAS, 135, 345
Rees, M. J. 1978, MNRAS, 184, 61

Rottmann, H., Mack, K.-H., Klein, U., \& Wielebinski, R. 1996, A\&A, 309, 19

Rybicki, G. B., \& Lightman, A. P. 1979, Radiative processes in astrophysic (New York: John Wiley \& Sons)

Ryle, M., \& Longair, M. S. 1967, MNRAS, 136, 123

Sarazin, C. L., \& Wise, M. W. 1993, ApJ, 411, 55

Sazonov, S. Yu., \& Sunyaev, R. A. 2003, A\&A, 399, 505

Scheuer, P. A. G. 1974, MNRAS, 166, 513

Sparks, W. B., Fraix-Burnet, D., Macchetto, F., \& Owen, F. N. 1992 Nature, 355, 804

Sunyaev, R. A. 1982, Sov. Astron. Lett., 8, 175

Sunyaev, R. A., \& Churazov, E. M. 1998, MNRAS, 297, 1279

Tonry, J. L. 1991, ApJ, 373, L1

van der Laan, H. 1963, MNRAS, 126, 535

Wise, M. W., \& Sarazin, C. L. 1992, ApJ, 395, 387 